\title{
Wavelength-Tunable Single-Mode Microlasers Based on Photoresponsive Pitch Modulation of Liquid Crystals for Information Encryption
}

\author{
Fa-Feng Xu $\mathbb{D}^{1,2}$ Zhong-Liang Gong $\mathbb{D}^{1,2}$ Yu-Wu Zhong $\mathbb{D}^{1,2}$ Jiannian Yao, ${ }^{1,2}$ \\ and Yong Sheng Zhao ${ }^{1,2}$ \\ ${ }^{1}$ Key Laboratory of Photochemistry, Institute of Chemistry, Chinese Academy of Sciences, Beijing 100190, China \\ ${ }^{2}$ University of Chinese Academy of Sciences, Beijing 100049, China \\ Correspondence should be addressed to Yong Sheng Zhao; yszhao@iccas.ac.cn
}

Received 23 June 2020; Accepted 3 November 2020; Published 2 December 2020

Copyright (c) 2020 Fa-Feng Xu et al. Exclusive Licensee Science and Technology Review Publishing House. Distributed under a Creative Commons Attribution License (CC BY 4.0).

\begin{abstract}
Information encryption and decryption have attracted particular attention; however, the applications are frequently restricted by limited coding capacity due to the indistinguishable broad photoluminescence band of conventional stimuli-responsive fluorescent materials. Here, we present a concept of confidential information encryption with photoresponsive liquid crystal (LC) lasing materials, which were used to fabricate ordered microlaser arrays through a microtemplate-assisted inkjet printing method. LC microlasers exhibit narrow-bandwidth single-mode emissions, and the wavelength of LC microlasers was reversibly modulated based on the optical isomerization of the chiral dopant in LCs. On this basis, we demonstrate phototunable information authentication on LC microlaser arrays using the wavelength of LC microlasers as primary codes. These results provide enlightenment for the implementation of microlaser-based cryptographic primitives for information encryption and anticounterfeiting applications.
\end{abstract}

\section{Introduction}

Information encryption and decryption have attracted tremendous interest for tightly protecting significant information, offering many advanced applications ranging from high-throughput data storage to secure communication $[1,2]$. The ever-increasing demands for high-level information security call for appropriate primitive systems capable of not only convenient information encryption but also efficient data authentication. Stimuli-responsive fluorescent materials have found their successful applications as data recording and document encryption systems owing to their tactfully changed luminescent outputs in response to external stimuli [3-5], which prevent the secret information from being stolen, mimicked, or forged. To date, various smart luminescent materials, including semiconductor nanocrystals $[6,7]$, dyes [8], lanthanide-doped nanoparticles $[9,10]$, carbon dots [11, 12], and transition-metal complexes $[13,14]$, have been explored. However, spontaneous emission from such materials usually produces broad photolumi- nescence (PL) bands, which are prone to be overlapped and result in the limited coding capacity [15-17]. Stimulated emission, which can generate discrete narrow lasing signals for easily distinguishable readout $[18,19]$, shows greater potential in the security field than conventional spontaneous emission [20-22]. Therefore, exploiting appropriate lasing systems as cryptographic primitives is of great significance for the implementation of high-security information encryption and decryption.

Liquid crystal (LC) materials with natural photonic crystal superstructures and excellent doping flexibility are promising candidate for the development of narrowbandwidth lasers [23, 24], which allow for potential encryption applications as cryptographic primitives. Moreover, as a flexible organic lasing system [25-27], LCs possess outstanding processability and can be fabricated into ordered microlaser arrays [28-30], affording an opportunity for high-throughput parallel encryption. Further, the helical pitch of LCs and corresponding wavelength of LC lasers can sensitively respond to external stimuli, such as thermal 


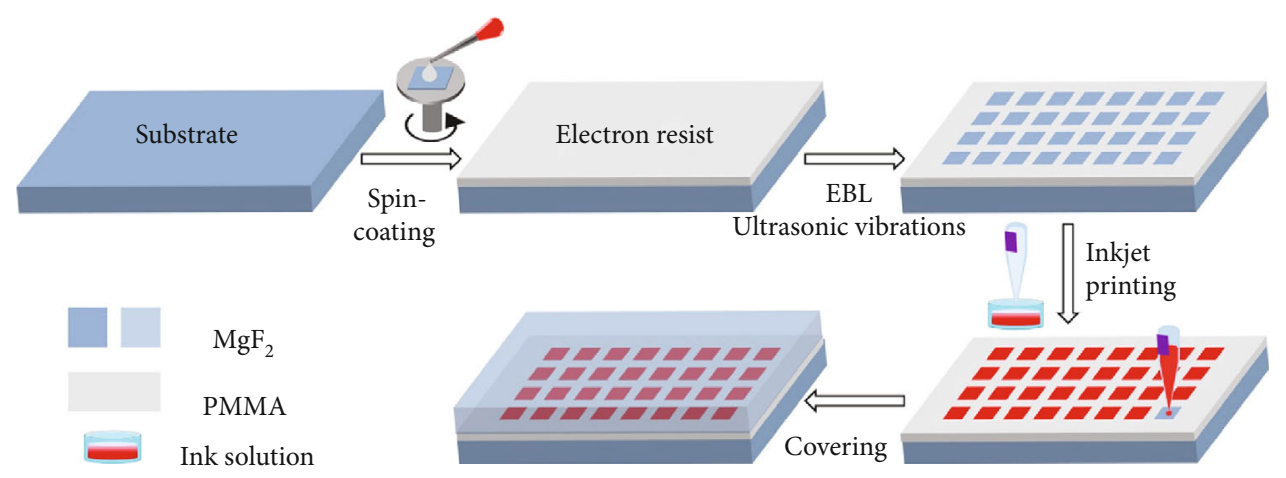

(a)

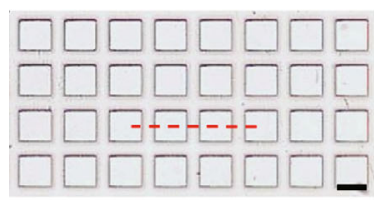

(b)

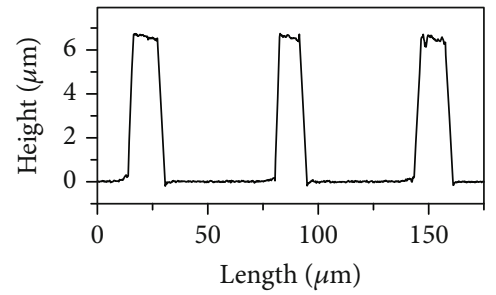

(c)

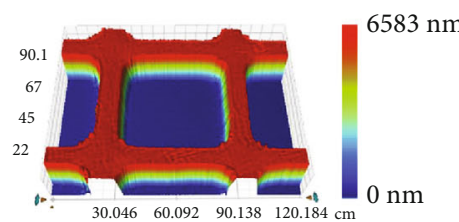

(d)

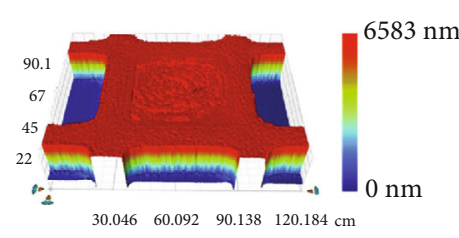

(e)

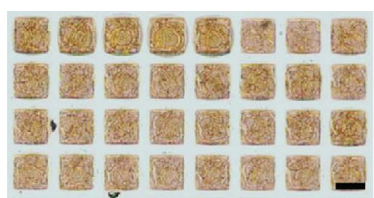

(f)

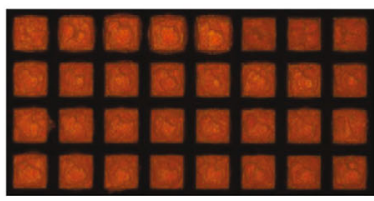

(g)

FIGURE 1: Fabrication procedures of a LC microlaser array. (a) Schematic diagram for the fabrication process of the LC microlaser array. (b) Optical microscopy image of polymeric microtemplates. (c) Plot of the height $v s$. the length of the microtemplates (marked as the dashed red line in (b)) by a step profiler. Scale bar: $50 \mu \mathrm{m}$. Three-dimensional profiles of a microtemplate filled (d) without and (e) with LC-contained ink solutions by a contourgraph. (f) Bright-field and (g) dark-field microcopy images of the LC microlaser array. Scale bar: $50 \mu \mathrm{m}$.

stimuli [31, 32], electrical stimuli $[33,34]$, and mechanical stimuli [35], which facilitates the fulfillment of stimuliresponsive confidential encryption. Thus, external fieldresponsive LC lasing systems would be an ideal platform for confidential information protection.

In this work, we present a concept of confidential information encryption using photoresponsive LC lasing materials, which were used to fabricate ordered microlaser arrays through a microtemplate-assisted inkjet printing method. Single-mode LC microlasers were obtained with LC superstructures serving as distributed feedback cavities and embedded luminescent dye providing optical gain. Optically controlled isomerization of chiral dopants regulated the helical pitch of LCs and permitted reversible modulation of the wavelength of LC microlasers. On this basis, we demonstrate phototunable information encryption and authentication on LC microlaser arrays using the wavelength of LC microlasers as primary code. These results open a new avenue for microlaser-based information encryption and will stimulate fascinating cryptographic applications.

\section{Results}

LC microlaser arrays were prepared by precisely depositing LC-contained ink solutions into polymeric microtemplates in regular alignment on substrates according to the predesigned digital patterns. As schematically illustrated in Figure 1(a), the fabrication procedure includes the preparation process of the polymeric microtemplates by etching the spin-coated film of the electron resist (polymethyl methacrylate (PMMA)) on the $\mathrm{MgF}_{2}$ substrate via electron beam lithography (EBL) that is a robust and effective technique for the production of microdevices, the printing process of depositing LC-contained ink solutions into the as-prepared microtemplates assisted by ultrasonic vibration, which is widely applied in the field of microelectronics, and the encapsulation process of covering another $\mathrm{MgF}_{2}$ wafer onto the ink-filled microtemplates. The as-fabricated polymeric microtemplates with microscale dimensions and depths (Figure 1(b)-(d)) can tightly confine the deposited ink solutions. The deposited ink solutions perfectly filled the microtemplates to produce individual microunits (Figure 1(e)), which can be precisely controlled by altering the vibration strength in the printing process (Fig. S1; see Materials and Methods). The structural parameter of the microunits, including shape (Fig. S2) and size (Fig. S3), can be rationally modulated by varying the microtemplates with the same parameters. The fabricated microunits were regularly patterned into an ordered emissive array (Figure 1(f) and (g)) according to the well-organized geometry of the microtemplates, which 


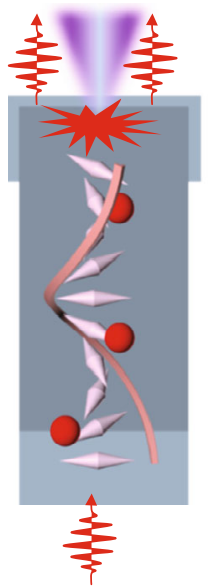

(a)

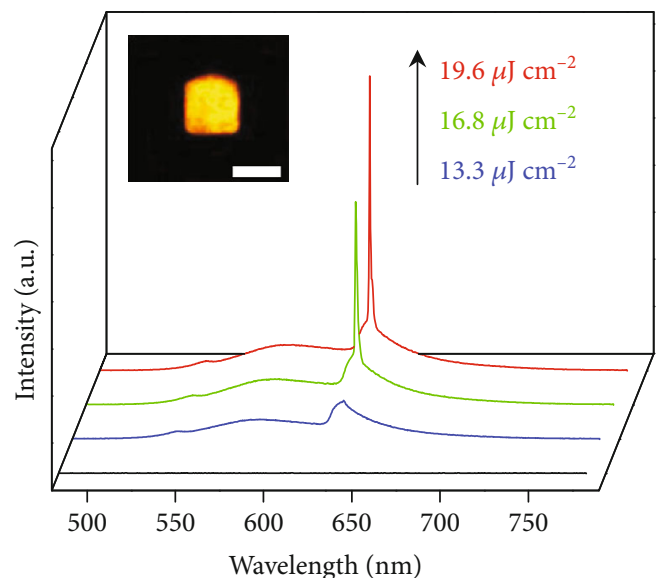

(c)
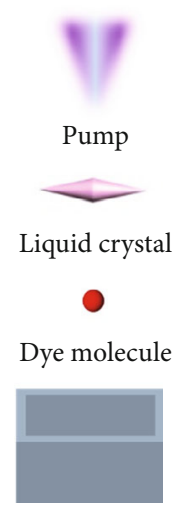

Microtemplate
Dye molecule

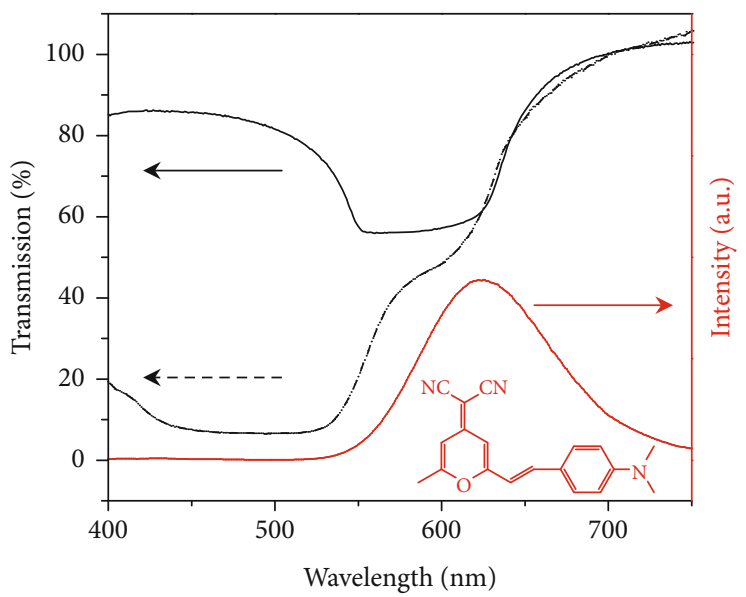

(b)

$$
\begin{aligned}
- & \text { CLCs } \\
--- & \text { Dye-doped } \\
& \text { CLCs }
\end{aligned}
$$

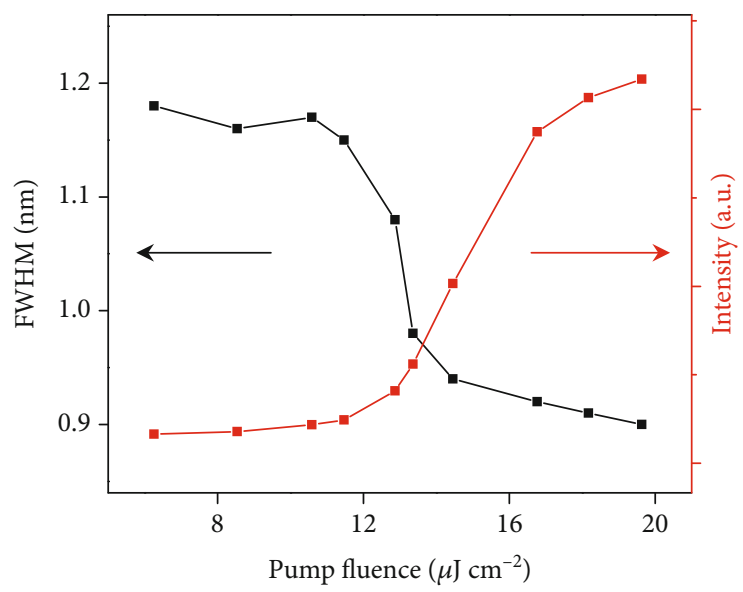

(d)

FIGURE 2: LC lasing from the printed microunit. (a) Sketch of an individual LC microlaser. (b) Transmission spectra of the CLCs doped with and without the dye and PL spectrum of the dye in ethanol. The concentration of the dye is $0.01 \mathrm{mM}$. Inset: the molecular structure of the dye. (c) PL spectra of an individual microunit under different pump fluences. Inset: PL image of the corresponding microunit. Scale bar: $50 \mu \mathrm{m}$. (d) Plots of the PL peak intensity and the FWHM of the microunit $v s$. the pump fluence.

serve as potential microlaser-based cryptographic primitives for information encryption applications.

Upon pulsed illumination, effective lasing from the asfabricated microunits would be expected with LC superstructures serving as photonic crystal cavities and embedded dyes providing optical gains, as depicted in Figure 2(a). In the microunits, through introducing chiral dopants into the LC matrix, cholesteric LC (CLC) superstructures were obtained since their pitches were captured and recorded in SEM images (Fig. S4) by using the UV curing method [36], which can serve as photonic crystal cavities to support laser oscillations at the band edges [37]. It should be noted that there is no alignment treatment of the substrate adopted here as it has been demonstrated not necessarily needed [28] and the formation of helical structures of CLC in the microunits probably can be ascribed to the induction of polymeric microtemplates [30]. The luminescent dye, 4-(dicyano-
methylene)-2-methyl-6-(4-dimethylaminostyryl)-4H-pyran (Fig. S5), with PL emissions matching the long-wavelength band edge of the CLCs (Figure 2(b)), was doped into the LC solutions, which did not lead to obvious shift of the band edge and thus ensures lasing at the designed wavelength.

When the as-fabricated microunit was uniformly excited by a pulsed laser beam $(490 \mathrm{~nm}, \sim 150 \mathrm{fs}$, and repetition rate of $1 \mathrm{kHz}$ ) in a custom microphotoluminescence system (Fig. S6), evidently modulated PL spectra of the microunit were observed, with much higher PL intensities at the band edges than that inside the bandgap (Fig. S7). Such unique modulation of PL emissions can be attributed to the fluctuation of density of optical states (DOS) at different positions of photonic bands of the CLCs [38], and the resulted higher DOS at band edges can favor the generation of lowthreshold laser emissions [39]. With increasing pump fluence, the PL intensities of the peak at $\sim 637 \mathrm{~nm}$, where the 
long-wavelength band edge of the CLCs locates, were dramatically amplified (Figure 2(c)). The plot of the corresponding PL peak intensity as a function of the pump fluence exhibited a clear knee behavior characteristic at the threshold of $\sim 13.2 \mu \mathrm{J} \mathrm{cm}^{-2}$ (Figure 2(d)), which confirmed the lasing action of the microunit. Above the onset power, the fullwidth at half-maximum (FWHM) of the PL peak sharply narrowed down to $\sim 0.9 \mathrm{~nm}\left(19.6 \mu \mathrm{J} \mathrm{cm}^{-2}\right)$, which revealed a microcavity effect with a quality factor of $\sim 703$ (defined as $\lambda / F W H M)$. Such narrow PL peaks are in stark contrast with broadband PL peaks of the dye in tradition matrix, which undergo spontaneous emission mechanisms (Fig. S8). Note that the experimental result of narrow peaks based on obvious broadband fluorescence backgrounds reflects that the stimulated emission merely dominates due to the good arrangement of LCs induced by microtemplates, which can be further improved via alignment treatment thus excellent lasing emission with flat backgrounds would be expected $[23,29]$. Single-mode lasing from the microunit was obtained due to the spatially modulated refractive index from CLC superstructures, which can be easily distinguished without confusion [40-42], and thus benefits the acquisition of lasing wavelength-based information encryption.

The lasing wavelength of the as-fabricated microunits can be effectively modulated through varying the lattice constant of the one-dimensional photonic crystal superstructures of the CLCs (the helical pitch), as the pitch determines the position of the photonic band and thus affects the resonant band edge mode [43]. The photoisomerization of chiral dopants (CDs) in LC matrixes has been demonstrated an effective scheme to tune the LC pitch [44-46]. In this work, an azobenzene chiral molecule [47] (CD-1) with large helical twisting power for highly sensitive photoresponsibility was adopted for its potential in achieving optically tunable LC lasers. Under UV/Vis irradiation, this molecule undergoes reversible trans-cis isomerization (Figure 3(a) and Fig. S9), leading to the modulation of the LC pitch and thus the shift of the corresponding photonic band edges. Efficient pitch modulation was verified by the transmission spectra of these photoresponsive CD-doped LCs, where obvious shift of the photonic band edge was observed under temporal UV (365 nm) illumination (Figure 3(b)). Consequently, the lasing wavelength of the LC microunit was effectively tuned to the bluer position $(601 \mathrm{~nm}$ ) (Figure 3(c)). Under the exposure to a visible light $(532 \mathrm{~nm})$, the lasing wavelength of the microunit was recovered $(637 \mathrm{~nm})$ due to the trans-isomerization of CD-1. In this way, the lasing wavelength of the microunit can be reversibly switched from $637 \mathrm{~nm}$ to $601 \mathrm{~nm}$ by altering the $365 \mathrm{~nm}$ and $532 \mathrm{~nm}$ light irradiations. The two lasing wavelengths remain nearly unchanged after six cycles (Figure 3(d)), demonstrating good reproducibility and tolerance with low device fatigue. It is worth noting that apart from the photoresponsive modulation, lasing wavelength could also move back to longer-wavelength position by itself after about 9 hours (Fig. S10) since the photoresponsive molecule $\mathrm{CD}-1$ can undergo thermal relaxation to a more stable trans state [47], which, however, is too long for conceptual and practical information encryption applications. Moreover, the wavelength-switchable lasing behavior of LC microunits can be maintained in several days owing to the well capsulation of the $\mathrm{MgF}_{2}$ wafers (Fig. S11), which verifies the good durability of LC microlasers in ambient condition. This optically controlled modulation of the lasing wavelength of the microunit affords an efficient authentication method for confidential information encryption.

As a proof-of-concept encryption application, we exploited LC microlasers as cryptographic primitives for information encryption, with their wavelengths representing binary codes to encrypt meaningful information. Remote and relatively fast optical modulation, instead of 9-hourperiod thermal relaxation modulation of lasing wavelength, was selected as the mechanism for the demonstration of minute-level confidential encryption and decryption. According to the ASCII binary codes, an arbitrary character can be translated into a standard 8-bit binary code sequence, e.g., "01010101" for the capital letter "U." Thus, specific raw information composed of a number of English characters, for instance, "UCAS" (acronym of University of Chinese Academy of Sciences), can be effectively encrypted in a series of LC microlasers, with two of their lasing wavelengths representing " 0 " and " 1 ," respectively (Figure 4(a)). In this work, two kinds of inks, ink 1 and ink 2, were prepared by incorporating photoresponsive and photo-nonresponsive chiral dopants into LC matrixes, respectively (Figure 4(b)). Information encryption was carried out by encoding the raw information in an $8 \times 4 \mathrm{LC}$ microlaser array via inkjet printing (Figure 4(c)). The bright-field photograph of the microlaser array suggests that the information is efficiently encrypted, despite the fact that these patterned microlasers were prepared with different inks. Upon an integral excitation (Fig. S12), laser emissions from individual microunits were generated, which are not visually distinguishable (Figure 4(d)), thus ensuring high security of the protected information. The initial wavelengths $(637 \mathrm{~nm})$ of those LC microlasers at specific positions shifted to bluer ones $(601 \mathrm{~nm})$ after UV illumination (Fig. S13), revealing the secret information of "UCAS" and this information was rehidden in the microlaser array after the illumination of a visible light, which demonstrates a prototype of confidential information encryption and authentication. These results manifest the applicability of wavelength-tunable LC microlasers as cryptographic primitives for high-security information protection.

\section{Discussion}

In conclusion, we have demonstrated confidential information encryption and authentication with phototunable LC microlaser arrays. The LC microlaser arrays were effectively fabricated via a microtemplate-assisted inkjet printing method, where single-mode lasing emissions from individual microunits were obtained. The photoisomerization of CDs permits the variation of pitches of LC superstructures and thus the modulation of the wavelength of LC microlasers. On this basis, we achieved high-security information encryption and authentication on LC microlaser arrays using the wavelength of the LC microlasers as primary codes. We postulate these results would provide valuable enlightenment to 


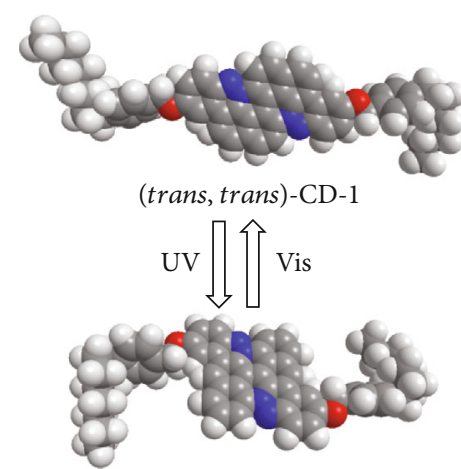

(cis, cis)-CD-1

(a)

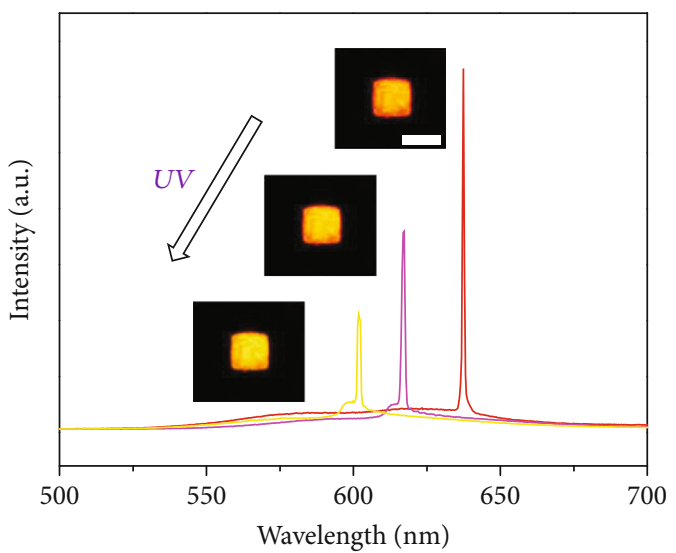

(c)

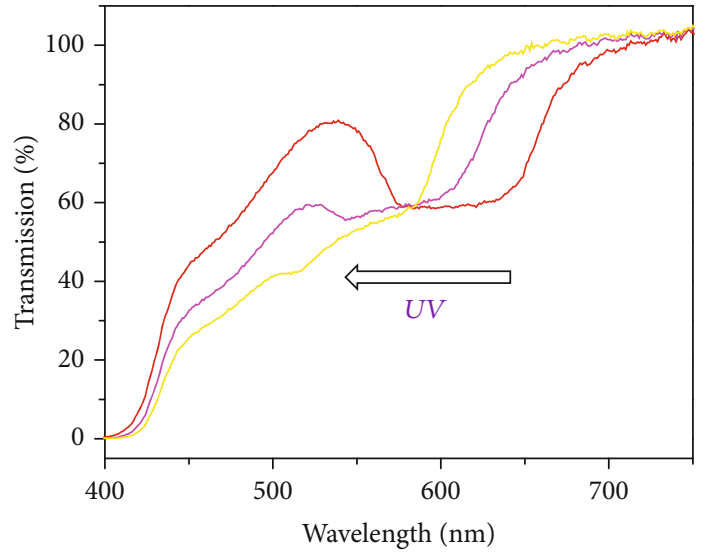

(b)

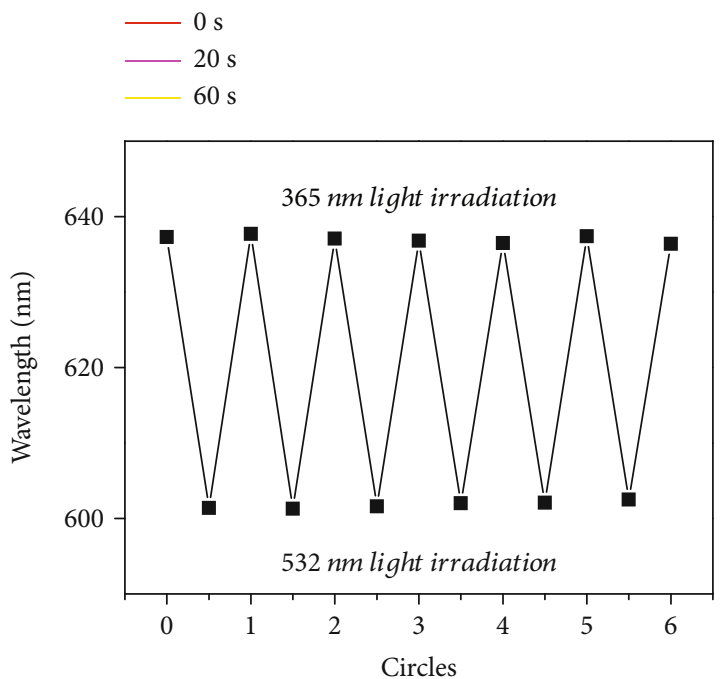

(d)

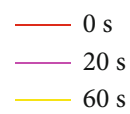

Figure 3: Phototunable lasing of the microunit. (a) Schematic illustration of the phototunable trans-cis isomerization process of CD-1. (b) Transmission spectra of CD-doped LCs under UV $\left(365 \mathrm{~nm}, 20 \mathrm{~mW} \mathrm{~cm}^{-2}\right.$ ) illumination with $0 \mathrm{~s}, 20 \mathrm{~s}$, and $60 \mathrm{~s}$. (c) PL spectra of the microunit under temporal UV illumination. Insets: PL images of the corresponding microunit. Scale bar: $50 \mu \mathrm{m}$. (d) Plot of the lasing wavelength $(637 \mathrm{~nm}$ and $601 \mathrm{~nm})$ against the switching cycles. In each cycle, the microunit is illuminated by a UV light (365 $\mathrm{nm}$, $20 \mathrm{~mW} \mathrm{~cm}^{-2}$ ) for $60 \mathrm{~s}$ and a visible light $\left(532 \mathrm{~nm}, 20 \mathrm{~mW} \mathrm{~cm}^{-2}\right)$ for $2 \mathrm{~m}$.

the development of wavelength-tunable microlaser-based information security and anticounterfeiting applications.

\section{Materials and Methods}

4.1. Materials. The liquid crystal (LC) (I32-010E-425) was purchased from Shijiazhuang Slichem Display Material Co. Ltd. and used as received without further treatment. 4-(Dicyanomethylene)-2-methyl-6-(4-dimethylaminostyryl)$4 \mathrm{H}$-pyran (98\%) was purchased from Sigma-Aldrich Co. and purified by sublimation in nitrogen atmosphere. The chiral dopant (CD) of CD-1 was synthesized according to Ref. [46]. 2-Methyl-1,4-phenylene bis(4-((6-(acryloyloxy)hexyl)oxy)benzoate) $(\mathrm{C} 6 \mathrm{M})$ as a photopolymerizable monomer and 2,2-dimethoxy-2-phenylacetophenone (Irgacure 651) as a photoinitiator for UV curing to capture LC pitches were purchased from Beijing Innochem Science \& Technology Co. Ltd. Araldite 506 epoxy resin was purchased from SigmaAldrich Co. The chiral dopant of CD-2 (S811) was purchased from Shijiazhuang Slichem Display Material Co. Ltd. and purified by sublimation in nitrogen atmosphere.

4.2. Fabrication of Liquid Crystal Microlaser Arrays. The polymeric microtemplates were prepared by etching polymeric films on substrates via electron beam lithography (EBL). In this work, $\mathrm{MgF}_{2}$ wafers were chosen as the substrates because of their low reflective index (1.38), which can reduce optical loss from the substrates and lower the threshold of laser emissions. The polymeric films were obtained by spin coating a poly(methyl methacrylate) 


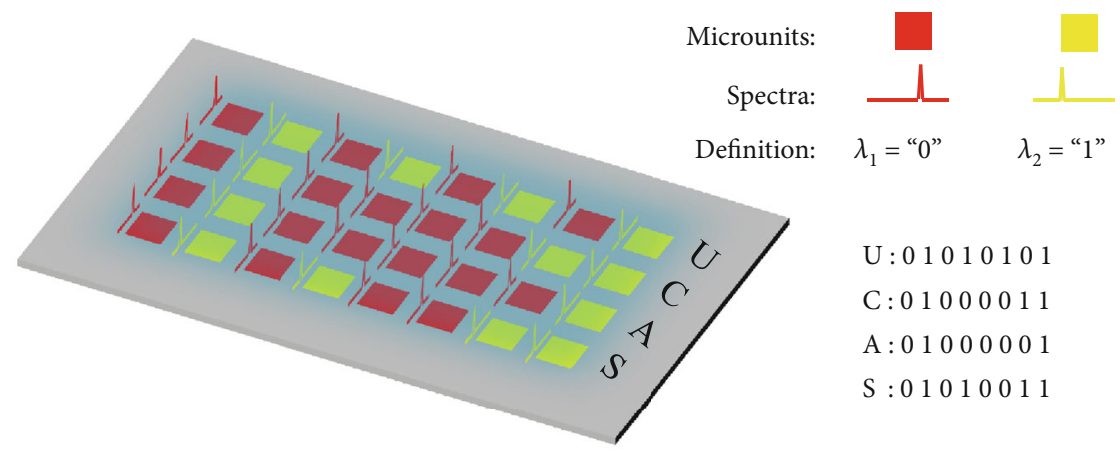

(a)

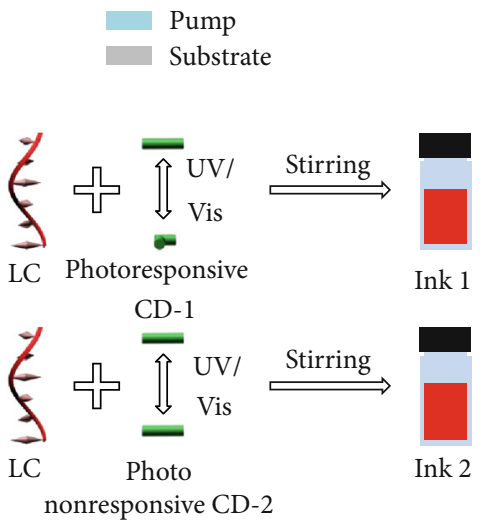

(b)

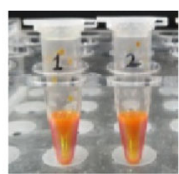

Dye-doped LC ink solutions

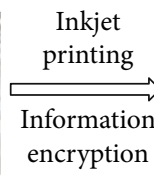

Information
encryption

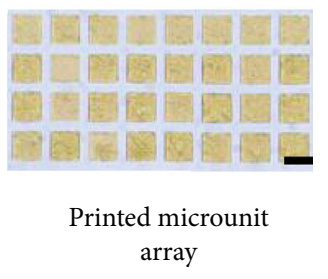

(c)

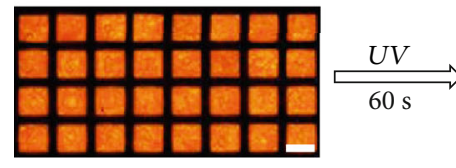

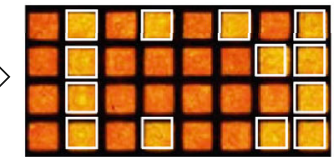

01010101

01000011

01000001

01010011
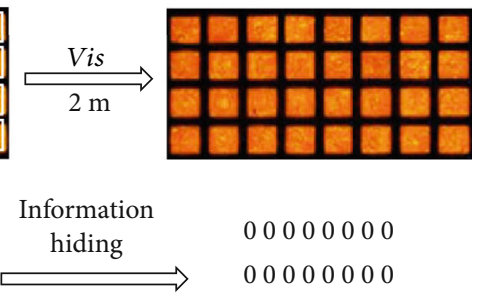

00000000

00000000

00000000

00000000

(d)

FIGURE 4: Confidential information encryption and authentication based on a phototunable LC microlaser array. (a) Concept of information encryption with patterned LC microlasers serving as cryptographic primitives. (b) Schematic illustration for the preparation process of chiral dopant-doped LC inks. Ink 1 and ink 2 are prepared by dissolving chiral dopants of photoresponsive CD-1 and photo-nonresponsive CD-2 into the LC matrix, respectively. (c) Information encryption process, which is carried out by encoding the raw information in a LC microlaser array via inkjet printing. Scale bar: $50 \mu \mathrm{m}$. (d) Information authentication and hiding processes on a LC microlaser array. The LC microlasers, whose wavelengths represent the binary code of " 1 ," are depicted as the white squares in the middle panel. Scale bar: $50 \mu \mathrm{m}$.

(PMMA) solution (20 wt\%, in chlorobenzene) on the pretreated (oxygen plasma, $5 \mathrm{~min}$ ) substrates at $1200 \mathrm{rpm}$ for $1 \mathrm{~min}$ and baked on a heating stage at $180^{\circ} \mathrm{C}$ for $5 \mathrm{~min}$ to eliminate the solvent. Then, electron beam exposures were carried out by an e-beam writer (ELPHY Quantum; Raith $\mathrm{GmbH}$, Germany) at $30 \mathrm{kV}$ with the beam-spot size of $3.0 \mathrm{~nm}$, the beam current of $120 \mathrm{pA}$, and the patterning dose of $0.5 \mathrm{mC} / \mathrm{cm}^{2}$. Finally, the sample was developed in the mixed solution of methyl isobutyl ketone and isopropyl alcohol $(1: 3)$ for $30 \mathrm{~s}$.

The LC-filled microunits were obtained by depositing inks into the polymeric microtemplates using a GIX $^{\mathrm{TM}}$ Micro- plotter $^{\mathrm{TM}}$ II from SonoPlot Inc. The cholesteric LC inks (ink 1 and ink 2) were prepared by dissolving $15 \mathrm{mg}$ of CD-1 and $25 \mathrm{mg}$ of CD-2 (S811) in the LC (I32-010E-425) matrix, respectively. The printing process included imbibing ink solutions with a hollow glass needle via capillary action and depositing the solutions into prefabricated microtemplates assisted by ultrasonic vibration. By adjusting the ultrasonic vibration strength, ink solution perfectly filled microunits were obtained.

The microunit laser arrays were fabricated by sequentially depositing luminescent inks into polymeric microtemplates on the substrate according to predesigned digital 
patterns. The red-emissive inks were prepared by dissolving $4.5 \mathrm{mg}$ each of the dye in $300 \mathrm{mg}$ of LC solution (1.5 wt\%). The inks (ink 1 and ink 2) were printed into the microtemplates at required positions to produce $8 \times 4$ microlaser arrays. Before printing, the glass needle was cleaned by washing (dichloromethane) to avoid ink contamination.

4.3. Characterization. The morphology of the polymeric microtemplates was examined by a step profiler (Bruker Dektak XT) and a contourgraph (Bruker Contour GT-K1). The superstructure pitch characteristics were examined with scanning electron microscopy (Hitachi SU8010). The absorption and fluorescence spectra were measured by a UV-visible spectrometer (Perkin-Elmer Lambda 35) and a fluorescent spectrometer (Hitachi F-7000), respectively. Bright-field and fluorescence microscopy images were taken using an inverted fluorescence microscope (Nikon Ti-U) by exciting the samples with the halogen and mercury lamps, respectively. The optically pumped lasing measurements for individual microunits were carried out on a custom microphotoluminescence system. The excitation pulses $(490 \mathrm{~nm})$ were generated from an optical parametric amplifier (Light Conversion TOPAS) pumped by a regenerative amplifier (Spectra Physics, $800 \mathrm{~nm}, 150 \mathrm{fs}, 1 \mathrm{kHz}$ ), which was in turn seeded by a mode-locked Ti : sapphire laser (Mai Tai, Spectra Physics, $800 \mathrm{~nm}, 150 \mathrm{fs}, 80 \mathrm{MHz}$ ). The excitation laser was filtered with a $500 \mathrm{~nm}$ short-pass filter and then focused down to a $20 \mu \mathrm{m}$ diameter spot through an objective lens (Nikon CFLU Plan, $\times 20$, N.A. $=0.5$ ) as a nearly uniform pump source. The power at the input was altered by a neutral density filter. The emissions from the individual microunits were collected by the same objective with a back-scattering configuration and analyzed by the spectrometer after removing the excitation beam with a $500 \mathrm{~nm}$ long-pass filter.

\section{Conflicts of Interest}

The authors declare that there is no conflict of interest regarding the publication of this article.

\section{Authors' Contributions}

Y.S.Z. conceived the original concept. Y.S.Z. and J.Y. supervised the project. F.F.X. and Z.G. designed the experiments and prepared the materials. F.F.X. performed the optical measurements. F.F.X., Y.-W.Z., and Y.S.Z. analyzed the data and wrote the paper. All authors discussed the results and commented on the manuscript.

\section{Acknowledgments}

This work was supported financially by the Ministry of Science and Technology of China (Grant No. 2017YFA0204502) and the National Natural Science Foundation of China (Grant Nos. 21533013 and 21790364).

\section{Supplementary Materials}

Fig. S1: three-dimensional profiles of the microunits fabricated under different vibration strengths. Fig. S2: bright- field and photoluminescence microscopy images of the microunits with different shapes. Fig. S3: bright-field and photoluminescence microscopy images of the microunits with different sizes. Fig. S4: superstructure pitch characterization of the liquid crystals. Fig. S5: spectral data of the laser dye. Fig. S6: schematic demonstration of the experimental setup for lasing characterization. Fig. S7: photoluminescence spectra of the microunit under different pump fluences. Fig. S8: photoluminescence spectra of the dye in the microunit filled with epoxy resin. Fig. S9: molecular structure and absorption spectra of the chiral dopant under UV illumination. Fig. S10: the plot of the wavelength of the liquid crystal microlaser vs. the time under dark condition. Fig. S11: the plot of the wavelength modulation of the liquid crystal microlaser vs. the time. Fig. S12: schematic illustration of the experimental approach for the integral excitation of the microunit array. Fig. S13: photoluminescence spectra of the microunit array shown in Figure $4(d)$. (Supplementary Materials)

\section{References}

[1] T. Sarkar, K. Selvakumar, L. Motiei, and D. Margulies, "Message in a molecule," Nature Communications, vol. 7, no. 1 , article $11374,2016$.

[2] E. Waks, K. Inoue, C. Santori et al., "Quantum cryptography with a photon turnstile," Nature, vol. 420, no. 6917, p. 762, 2002.

[3] Z. Chi, X. Zhang, B. Xu et al., "Recent advances in organic mechanofluorochromic materials," Chemical Society Reviews, vol. 41, no. 10, pp. 3878-3896, 2012.

[4] A. Kishimura, T. Yamashita, K. Yamaguchi, and T. Aida, "Rewritable phosphorescent paper by the control of competing kinetic and thermodynamic self-assembling events," Nature Materials, vol. 4, no. 7, pp. 546-549, 2005.

[5] K. Li, Y. Xiang, X. Wang et al., "Reversible photochromic system based on rhodamine B salicylaldehyde hydrazone metal complex," Journal of the American Chemical Society, vol. 136, no. 4, pp. 1643-1649, 2014.

[6] C. Zhang, B. Wang, W. Li et al., "Conversion of invisible metal-organic frameworks to luminescent perovskite nanocrystals for confidential information encryption and decryption," Nature Communications, vol. 8, no. 1, article 1138, 2017.

[7] Y. Wang, X. Li, S. Sreejith et al., "Photon driven transformation of cesium lead halide perovskites from few-monolayer nanoplatelets to bulk phase," Advanced Materials, vol. 28, no. 48, pp. 10637-10643, 2016.

[8] X. Luo, J. Li, C. Li et al., "Reversible switching of the emission of diphenyldibenzofulvenes by thermal and mechanical stimuli," Advanced Materials, vol. 23, no. 29, pp. 3261-3265, 2011.

[9] H. Dong, S. R. Du, X. Y. Zheng et al., "Lanthanide nanoparticles: from design toward bioimaging and therapy," Chemical Reviews, vol. 115, no. 19, pp. 10725-10815, 2015.

[10] X. Li, Y. Xie, B. Song et al., "A Stimuli-Responsive Smart Lanthanide Nanocomposite for Multidimensional Optical Recording and Encryption," Angewandte Chemie International Edition, vol. 56, no. 10, pp. 2689-2693, 2017.

[11] K. Jiang, Y. Wang, C. Cai, and H. Lin, "Conversion of Carbon Dots from Fluorescence to Ultralong Room-Temperature Phosphorescence by Heating for Security Applications," Advanced Materials, vol. 30, no. 26, article 1800783, 2018. 
[12] Q. Lou, S. Qu, P. Jing et al., "Water-triggered luminescent "nano-bombs" based on supra-(carbon nanodots)," Advanced Materials, vol. 27, no. 8, pp. 1389-1394, 2015.

[13] T. Mutai, H. Satou, and K. Araki, "Reproducible on-off switching of solid-state luminescence by controlling molecular packing through heat-mode interconversion," Nature Materials, vol. 4, no. 9, pp. 685-687, 2005.

[14] H. Sun, S. Liu, W. Lin et al., "Smart responsive phosphorescent materials for data recording and security protection," Nature Communications, vol. 5, no. 1, article 3601, 2014.

[15] H. Lee, J. Kim, H. Kim, J. Kim, and S. Kwon, "Colour-barcoded magnetic microparticles for multiplexed bioassays," Nature Materials, vol. 9, no. 9, pp. 745-749, 2010.

[16] J. Lee, P. W. Bisso, R. L. Srinivas, J. J. Kim, A. J. Swiston, and P. S. Doyle, "Universal process-inert encoding architecture for polymer microparticles," Nature Materials, vol. 13, no. 5, pp. 524-529, 2014.

[17] J. Li, C. Hou, C. Huang et al., "Boosting Circularly Polarized Luminescence of Organic Conjugated SystemsviaTwisted Intramolecular Charge Transfer," Research, vol. 2020, article 3839160, 10 pages, 2020.

[18] M. Schubert, A. Steude, P. Liehm et al., "Lasing within live cells containing intracellular optical microresonators for barcodetype cell tagging and tracking," Nano Letters, vol. 15, no. 8, pp. 5647-5652, 2015.

[19] Z. Gao, C. Wei, Y. Yan et al., "Covert photonic barcodes based on light controlled acidichromism in organic dye doped whispering-gallery-mode microdisks," Advanced Materials, vol. 29, no. 30, article 1701558, 2017.

[20] M. Karl, J. M. E. Glackin, M. Schubert et al., "Flexible and ultra-lightweight polymer membrane lasers," Nature Communications, vol. 9, no. 1, article 1525, 2018.

[21] Z. Gao, K. Wang, Y. Yan, J. Yao, and Y. S. Zhao, "Smart responsive organic microlasers with multiple emission states for high-security optical encryption," National Science Review, 2020.

[22] Y. Yao, Z. Gao, Y. Lv et al., "Heteroepitaxial growth of multiblock Ln-MOF microrods for photonic barcodes," Angewandte Chemie, International Edition, vol. 58, no. 39, pp. 13803-13807, 2019.

[23] H. Coles and S. Morris, "Liquid-crystal lasers," Nature Photonics, vol. 4, no. 10, pp. 676-685, 2010.

[24] F. F. Xu, Y. J. Li, Y. Lv et al., "Flat-Panel Laser Displays Based on Liquid Crystal Microlaser Arrays," CCS Chemistry, vol. 2, pp. 369-375, 2020.

[25] C. Zhang, C.-L. Zou, Y. Zhao et al., "Organic printed photonics: from microring lasers to integrated circuits," Science Advances, vol. 1, no. 8, article e1500257, 2015.

[26] J. Zhao, Y. Yan, Z. Gao et al., "Full-color laser displays based on organic printed microlaser arrays," Nature Communications, vol. 10, no. 1, p. 870, 2019.

[27] V. D. Ta, S. Yang, Y. Wang et al., "Multicolor lasing prints," Applied Physics Letters, vol. 107, no. 22, article 221103, 2015.

[28] D. J. Gardiner, W. K. Hsiao, S. M. Morris et al., "Printed photonic arrays from self-organized chiral nematic liquid crystals," Soft Matter, vol. 8, no. 39, p. 9977, 2012.

[29] S. M. Morris, P. J. Hands, S. Findeisen-Tandel, R. H. Cole, T. D. Wilkinson, and H. J. Coles, "Polychromatic liquid crystal laser arrays towards display applications," Optics Express, vol. 16, no. 23, pp. 18827-18837, 2008.
[30] G. Strangi, V. Barna, R. Caputo et al., "Color-tunable organic microcavity laser array using distributed feedback," Physical Review Letters, vol. 94, no. 6, article 063903, 2005.

[31] D. S. Wiersma and S. Cavalieri, "A temperature-tunable random laser," Nature, vol. 414, no. 6865, pp. 708-709, 2001.

[32] S. S. Lee, J. B. Kim, Y. H. Kim, and S.-H. Kim, "Wavelengthtunable and shape-reconfigurable photonic capsule resonators containing cholesteric liquid crystals," Science Advances, vol. 4, no. 6, article eaat8276, 2018.

[33] J. Xiang, A. Varanytsia, F. Minkowski et al., "Electrically tunable laser based on oblique heliconical cholesteric liquid crystal," Proceedings of the National Academy of Sciences of the United States of America, vol. 113, no. 46, pp. 12925-12928, 2016.

[34] M. Humar, M. Ravnik, S. Pajk, and I. Muševič, "Electrically tunable liquid crystal optical microresonators," Nature Photonics, vol. 3, no. 10, pp. 595-600, 2009.

[35] H. Finkelmann, S. T. Kim, A. Munoz, P. Palffy-Muhoray, and B. Taheri, "Tunable mirrorless lasing in cholesteric liquid crystalline elastomers," Advanced Materials, vol. 13, no. 14, pp. 1069-1072, 2001.

[36] W. Hu, H. Zhao, L. Song et al., "Electrically controllable selective reflection of chiral nematic liquid crystal/chiral ionic liquid composites," Advanced Materials, vol. 22, no. 4, pp. 468-472, 2010.

[37] P. J. W. Hands, D. J. Gardiner, S. M. Morris, C. Mowatt, T. D. Wilkinson, and H. J. Coles, "Band-edge and random lasing in paintable liquid crystal emulsions," Applied Physics Letters, vol. 98, no. 14, article 141102, 2011.

[38] T. K. Mavrogordatos, S. M. Morris, F. Castles et al., "Density of photon states in dye-doped chiral nematic liquid crystal cells in the presence of losses and gain," Physical Review E, vol. 86, no. 1, article 011705, 2012.

[39] Y. J. Liu, X. W. Sun, P. Shum et al., "Low-threshold and narrow-linewidth lasing from dye-doped holographic polymer-dispersed liquid crystal transmission gratings," Applied Physics Letters, vol. 88, no. 6, article 061107, 2006.

[40] C. Zhang, C.-L. Zou, H. Dong, Y. Yan, J. Yao, and Y. S. Zhao, "Dual-color single-mode lasing in axially coupled organic nanowire resonators," Science Advances, vol. 3, no. 7, article e1700225, 2017.

[41] W. Zhang, J. Yao, and Y. S. Zhao, "Organic micro/nanoscale lasers," Accounts of Chemical Research, vol. 49, no. 9, pp. 1691-1700, 2016.

[42] X. Lin, Y. Liu, K. Wang et al., "Hybrid Three-Dimensional Spiral $\mathrm{WSe}_{2}$ Plasmonic Structures for Highly Efficient Second-Order Nonlinear Parametric Processes," Research, vol. 2018, article 4164029, 8 pages, 2018.

[43] J. H. Choi, Y. S. No, J. P. So et al., "A high-resolution straingauge nanolaser," Nature Communications, vol. 7, no. 1, article 11569, 2016.

[44] P. V. Shibaev, R. L. Sanford, D. Chiappetta, V. Milner, A. Genack, and A. Bobrovsky, "Light controllable tuning and switching of lasing in chiral liquid crystals," Optics Express, vol. 13, no. 7, p. 2358, 2005.

[45] A. Chanishvili, G. Chilaya, G. Petriashvili et al., "Lasing in dye-doped cholesteric liquid crystals: two new tuning strategies," Advanced Materials, vol. 16, no. 910, pp. 791-795, 2004 . 
[46] J. Li, H. K. Bisoyi, S. Lin, J. Guo, and Q. Li, "1,2-Dithienyldicyanoethene-based, visible-light-driven, chiral fluorescent molecular switch: rewritable multimodal photonic devices," Angewandte Chemie, vol. 131, no. 45, pp. 16198-16202, 2019.

[47] J. Ma, Y. Li, T. White, A. Urbas, and Q. Li, "Light-driven nanoscale chiral molecular switch: reversible dynamic full range color phototuning," Chemical Communications, vol. 46, no. 20, pp. 3463-3465, 2010. 